\title{
Long noncoding RNA GAPLINC promotes gastric cancer cell proliferation by acting as a molecular sponge of miR-378 to modulate MAPKI expression
}

This article was published in the following Dove Press journal:

OncoTargets and Therapy

\section{Lingyun Diao ${ }^{1,2}$ \\ Shengying Wang ${ }^{2}$ \\ Zhiguang Sun'}

'Department of Gastroenterology, The First Clinical Medical School of Nanjing University of Chinese Medicine, Nanjing, People's Republic of China; ${ }^{2}$ Department of Gastroenterology, Xuzhou City Hospital of Traditional Chinese Medicine, Xuzhou, People's Republic of China
Correspondence: Zhiguang Sun Department of Gastroenterology, The First Clinical Medical School of Nanjing University of Chinese Medicine, No 138 Xianlin Avenue, 210023 Nanjing, Jiangsu, People's Republic of China

Tel +862586618472

Fax +862586518612

Email zhiguangsun_dr@sina.com
Background: Dysregulated long noncoding RNAs (lncRNAs) and microRNAs (miRNAs) play key roles in the development of human cancers. LncRNA GAPLINC has been reported to be increased in gastric cancer (GC) tissues.

Methods: Real-time PCR assays were used to measure expressions of GAPLINC, miR-378, and MAPK1 mRNA. Western blot assays were employed to examine MAPK1 protein expression. Cell proliferation and cell cycle were measured by CCK- 8 and propidium iodide-detection assays, respectively. The interaction between GAPLINC and miR-378 was confirmed by site-directed mutagenesis and luciferase assays. Luciferase assays were also used to study whether GAPLINC was able to act as a molecular sponge of miR-378 to modulate MAPK1 expression.

Results: The lncRNA GAPLINC expression was upregulated and positively correlated with MAPK1 expression in gastric cancer tissues and cells. Additionally, lncRNA GAPLINC promoted the expression of MAPK1 and the enhancement of GC cell proliferation and cell cycle progression by LncRNA GAPLINC was dependent on MAPK1 in vitro and in vivo. Consequently, we found that miR-378 expression was inversely correlated with GAPLINC expression in GC tissues and cells. miR-378 could directly bind to GAPLINC and decreased GAPLINC expression, thus reducing MAPK1 expression. Furthermore, overexpression of miR-378 inhibited MAPK1 expression, cell proliferation, and cell cycle progression of gastric cancer cells, while these effects were abrogated by upregulating lncRNA GAPLINC expression.

Conclusion: Taken together, IncRNA GAPLINC promotes gastric cancer cell proliferation by acting as a molecular sponge of miR-378 to modulate MAPK1 expression.

Keywords: GAPLINC, cell proliferation, miR-378, MAPK1, gastric cancer

\section{Introduction}

Gastric cancer (GC) is the fourth most common cancer and the second leading cause of cancer-related death worldwide. ${ }^{1}$ To facilitate early diagnosis and improve targeted therapy of GC, an in-depth understanding of molecular underpinnings of the disease is required..$^{2-4}$ Therefore, it is imperative to elucidate the regulatory network underlying gastric carcinogenesis to develop novel biomarkers for diagnosis and targeted therapy. ${ }^{5-7}$

Long noncoding RNAs (lncRNAs) are functional RNAs more than 200 nucleotides in length. ${ }^{8}$ Many lncRNAs regulate gene expression at several levels including transcription and posttranscriptional processing. ${ }^{8}$ In a recently described regulatory mechanism, lncRNAs behave as competing endogenous RNAs (ceRNAs), acting as molecular sponges of microRNAs (miRNAs) to derepress miRNA targets. ${ }^{9}$ For example, the IncRNA PVT1 functions as a ceRNA to regulate HIF-1 $\alpha$ expression by sponging the miR-186 in human GC. ${ }^{10}$ LncRNA CRNDE, a ceRNA of miR-145, 
is highly expressed in GC, and may be a potential target of antitumor therapy. ${ }^{11}$ These findings suggest the involvement of lncRNA GAPLINC in GC tumorigenesis.

Here, we investigated the effects of lncRNA GAPLINC on GC and the correlation between GAPLINC and MAPK1. We found that lncRNA GAPLINC was increased and positively correlated with MAPK1 expression in GC tumor tissues. Additionally, the enhancement of GC cell proliferation and cell cycle progression by lncRNA GAPLINC was dependent on MAPK1. Moreover, mechanistic analysis revealed that GAPLINC might function as a ceRNA to regulate the expression of MAPK1 via competitively binding miR378 , thus playing an oncogenic role in gastric carcinoma. Collectively, these data contribute to the understanding of the roles and molecular mechanisms of GAPLINC in GC progression.

\section{Materials and methods Clinical ethics}

Approval from Institutional Research Ethics Committee of Nanjing University of Chinese Medicine was obtained prior to the use of the clinical materials for research purposes. All patients provided their written informed consent to participate in this study.

\section{Tissue samples and cell lines}

We examined tumor and paired adjacent para-tumor tissues from 53 patients with GC. All tumor and paired adjacent para-tumor tissues were confirmed by experienced pathologists. The GC cell lines KATO III, HGC-27, and SNU-1 cells were purchased from Chinese Academy of Sciences (Shanghai, People's Republic of China). Cells were cultured in DMEM (Thermo Fisher Scientific, Waltham, MA, USA) supplemented with 10\% FBS (Thermo Fisher Scientific) in a humidified $5 \% \mathrm{CO}_{2}$ atmosphere at $37^{\circ} \mathrm{C}$.

\section{Cell transfection}

LncRNA GAPLINC sequence was purchased and subcloned into pCDNA3 plasmid from GENEWIZ (South Planfield, NJ, USA). miR-378 mimics or inhibitor or their respective controls were purchased from GenePharma (Shanghai, People's Republic of China). MAPK1 siRNA, GAPLINC siRNA, and control siRNAs were purchased from Thermo Fisher Scientific. Cells were transfected using transfection reagent Lipofectamine 2000 (Thermo Fisher Scientific) according to the manufacturer's protocol. They were harvested $24 \mathrm{~h}$ after transfection. For co-transfection, cells were initially transfected with miR-378 control, mimics, or inhibitor using Lipofectamine 2000 (Thermo Fisher Scientific), according to the manufacturer's instructions. Subsequently, the cells were transfected with MAPK1 siRNA, GAPLINC siRNA, or pCDNA-GAPLINC using Lipofectamine 2000.

\section{Cell proliferation and cell cycle analysis}

For analysis of cell proliferation, cells were seeded into 96-well plates at $4 \times 10^{3}$ cells $/$ well. Cells were incubated in $10 \%$ cell counting kit-8 (CCK-8; Dojindo, Kumamoto, Japan) and diluted in normal culture medium at $37^{\circ} \mathrm{C}$ until visual color conversion occurred. The proliferation rate was determined at $0,24,48$, and $72 \mathrm{~h}$ after transfection. The absorbance in each well was measured with OD $450 \mathrm{~nm}$. All experiments were performed in triplicate. Cell cycle analysis was performed in GC cells $48 \mathrm{~h}$ after transfection. Cells were harvested, washed twice with cold PBS, fixed in ice-cold 70\% ethanol, incubated with propidium iodide and RNase A, and then analyzed by FACS. All experiments were run in triplicate.

\section{Real-time PCR}

Total RNA was extracted from GC cells using the TRIzol method (Thermo Fisher Scientific). Reverse transcription was performed using reverse transcriptase kit (Takara, Otsu, Japan) according to the manufacturer's protocol. Real-time PCR was performed using an SYBR premix Ex Taq kit (Takara) on 7300 Real-Time PCR System (Thermo Fisher Scientific) according to the manufacturer's protocol. The miR-378 and U6 primer sequences used were obtained from GenePharma. The expression levels of U6 and GAPDH were used as controls, respectively. The data were analyzed by $2^{-\Delta \Delta \mathrm{Ct}}$ method.

\section{Western blot analysis}

The whole-cell protein was lysed on ice in RIPA (KenGEN, People's Republic of China) containing protease inhibitor cocktail (Thermo Fisher Scientific) and was quantified using a BCA Protein Assay Kit (Thermo Fisher Scientific). Quantified protein lysates were separated by an SDS-PAGE gel and transferred to PVDF membranes (EMD Millipore, Billerica, MA, USA). The membrane was blocked in 5\% nonfat milk and incubated with diluted antibodies against MAPK1 (Cell Signaling, Danvers, MA, USA) and GAPDH (Cell Signaling), followed by incubation with an HRPconjugated secondary antibody (Santa Cruz, Dallas, TX, USA). The PVDF membranes were washed and visualized using ECL chemiluminescence (Bio-Rad, Hercules, CA, USA). GAPDH was used as a control.

\section{Dual-luciferase reporter assay}

The fragments from GAPLINC containing the predicted hsamiR-378 binding site were synthesized and cloned into the pmiRGLO vector (GENEWIZ). The resulted vectors were 
called the reporter vector pmiRGLO-GAPLINC-wild type (pmiRGLO-GAPLINC-WT). The corresponding mutants were created by mutating the hsa-miR-378 seed region binding site, which were called the reporter vector pmiRGLOGAPLINC-mutated type (pmiRGLO-GAPLINC-MUT). miR-378 mimics or control were co-transfected with the reporter vectors containing either the targeting sequences or the corresponding mutants using transfection reagent Lipofectamine 2000 (Thermo Fisher Scientific) according to the manufacturer's protocol. After $48 \mathrm{~h}$ of transfection, the luciferase assay was performed using a Dual-Luciferase Reporter Assay System (Promega, Madison, WI, USA) according to the manufacturer's protocol.

\section{Xenograft tumor model}

The animal experiments were approved by the Committee on Animal Care of Nanjing University of Chinese Medicine and were conducted according to NIH Guidelines for the Care and Use of Laboratory Animals. All studies involving animals are reported in accordance with the ARRIVE guidelines for reporting experiments involving animals. Male nude mice (6 weeks old) were purchased from the Vital River Laboratories (Beijing, People's Republic of China) and randomly divided into three groups. GC cell line KATO III cells were transfected with control, pCDNA-GAPLINC, or pCDNA-GAPLINC + MAPK1 siRNA. Cells were cultured for $24 \mathrm{~h}$ before harvest for transplantation into the animals. Before injection, cells were pooled and mixed with matrigel (BD Biosciences, Franklin, NJ, USA). Then, $10^{5}$ cells were injected subcutaneously into the right flank of nude mice. Measurements were taken weekly, and tumor volumes were calculated using the formula $V=$ length $\times$ width ${ }^{\wedge} 2 / 2$. The animals were sacrificed 4 weeks after injection.

\section{Statistical analysis}

Data were expressed as the mean \pm SD of at least three independent experiments. Statistical analysis was carried out using GraphPad Prism 5 software (GraphPad Software, La Jolla, CA, USA). Student's $t$-test or ANOVA was performed to analyze the data. $P<0.05$ was considered statistically significant.

\section{Results \\ Expression of IncRNA GAPLINC was increased and positively correlated with MAPKI expression in clinical GC tissues}

Mao et al have suggested that lncRNA GAPLINC may be involved in MAPK signaling pathway by transcriptome analysis of miRNA-lncRNA-mRNA interactions in GC. ${ }^{12}$ Therefore, we examined the expression levels of lncRNA GAPLINC and MAPK1 in 53 pairs of human GC tissues and adjacent para-tumor tissues. Our results of real-time PCR assay revealed that compared with adjacent para-tumor tissues, IncRNA GAPLINC and MAPK1 mRNA were both upregulated in GC tumor tissues (Figure 1A and B). Furthermore, Pearson's correlation analysis showed a significant positive correlation between lncRNA GAPLINC and MAPK1 in GC tumor tissues ( $\mathrm{n}=30, r=0.41, P=0.0024$; Figure $1 \mathrm{C})$.

\section{LncRNA GAPLINC promoted the expression of MAPKI in GC cells}

To explore the effect of GAPLINC on MAPK1 expression of GC cells, we examined the expression levels of GAPLINC and MAPK1 in three human GC cell lines: KATO III, HGC-27, and SNU-1. The results confirmed a significant positive correlation between lncRNA GAPLINC and MAPK1 in GC cell lines (Figure 2A and B). Based on the results above, we furthered our research by silencing the
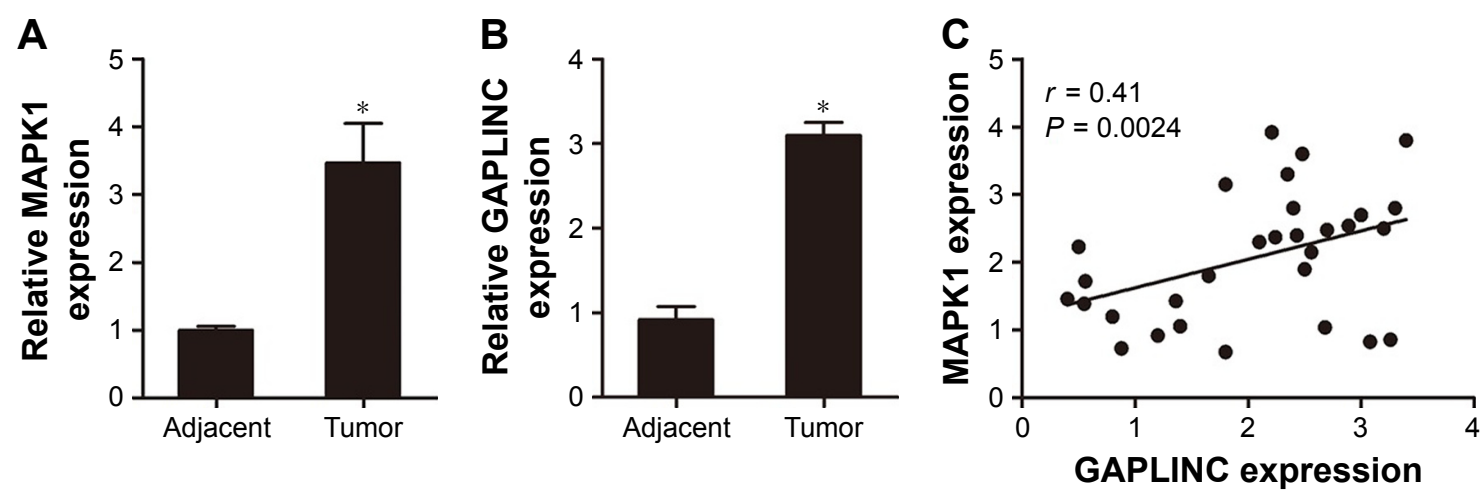

Figure I LncRNA GAPLINC expression was increased and positively correlated with MAPKI expression in clinical GC tissues. (A and B) Relative expression of MAPKI and IncRNA GAPLINC in GC tumor tissues and adjacent para-tumor tissues was analyzed by real-time PCR. Results are represented as the mean \pm SD. $n=53$, Student's $t$-test, $* P<0.05$. (C) Pearson's correlation analysis showed a significant positive correlation between IncRNA GAPLINC and MAPKI in GC tumor tissues (Spearman's correlation analysis, $\mathrm{n}=30, r=0.4 \mathrm{I}, \mathrm{P}=0.0024)$.

Abbreviations: IncRNA, long noncoding RNA; GC, gastric cancer. 

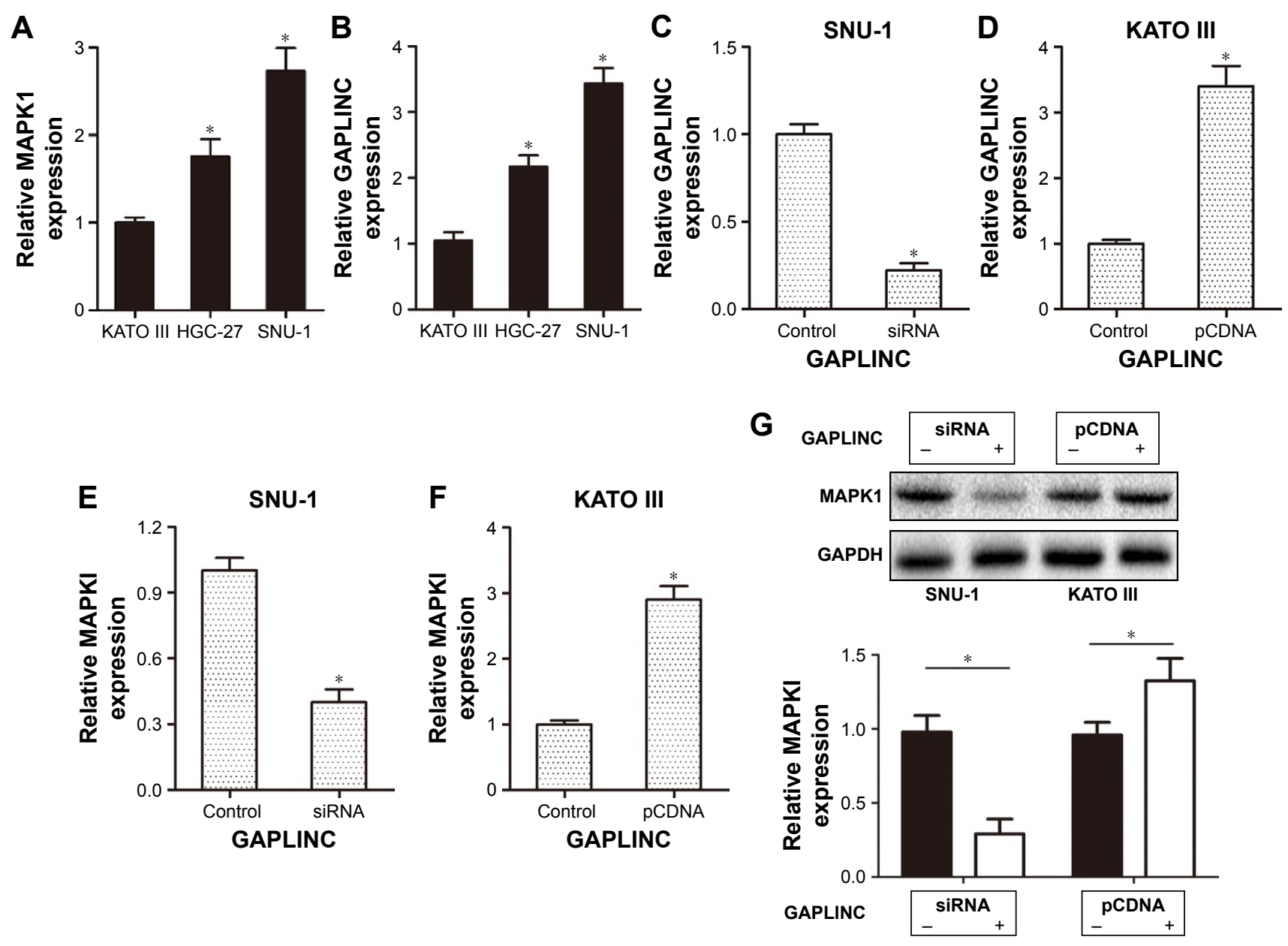

Figure 2 LncRNA GAPLINC promoted the expression of MAPKI in GC cells. (A and B) Relative GAPLINC and MAPKI expression was analyzed by real-time PCR in GC cell lines (KATO III, HGC-27, and SNU-I). Results are represented as the mean \pm SD. $n=4$, Student's $t$-test, $* P<0.05$. (C and D) Relative expression of GAPLINC was analyzed by real-time PCR in SNU-I after transient transfection of GAPLINC siRNA or KATO III after transient transfection of pCDNA-GAPLINC. Results are represented as the mean \pm SD. $n=3$, Student's $t$-test, ${ }^{*} P<0.05$. (E and $\mathbf{F}$ ) Relative expression of MAPKI was analyzed by real-time PCR in SNU-I after transient transfection of GAPLINC siRNA or KATO III after transient transfection of PCDNA-GAPLINC. Results are represented as the mean \pm SD. $n=3$, Student's $t$-test, $* P<0.05$. (G) Relative expression of MAPKI protein was analyzed by Western blotting in SNU-I after transient transfection of GAPLINC siRNA or KATO III after transient transfection of PCDNA-GAPLINC. Results are represented as the mean $\pm S D$. $n=3$, Student's $t$-test, $* p<0.05$.

Abbreviations: IncRNA, long noncoding RNA; GC, gastric cancer.

expression of GAPLINC in SNU-1 which has a relatively high GAPLINC expression and upregulating the expression of GAPLINC in KATO III which has a relatively low GAPLINC expression. The results suggested that GAPLINC expression in SNU-1 was clearly decreased after transfection with GAPLINC siRNA and GAPLINC expression in KATO III was obviously increased after transfection with pCDNA-GAPLINC compared with the control-transfected groups, respectively (Figure $2 \mathrm{C}$ and $\mathrm{D}$ ). Consequently, we examined MAPK1 mRNA and protein levels in GAPLINC siRNA-transfected SNU-1 cells and in pCDNA-GAPLINCtransfected KATO III cells using real-time PCR and Western blotting assays. The results showed that both MAPK1 mRNA and protein levels were decreased in GAPLINC siRNA-transfected SNU-1 cells and were increased in pCDNA-GAPLINC-transfected KATO III cells (Figure 2E-G).

\section{Enhancement of GC cell proliferation} and cell cycle progression by IncRNA GAPLINC was dependent on MAPK I

To study the effects of GAPLINC on proliferation and cell cycle progression of GC cells and whether these effects of GAPLINC are dependent on MAPK1, we transfected pCDNA-GAPLINC or pCDNA-GAPLINC + MAPK1 siRNA in KATO III cells. CCK-8 assay showed that GAPLINC overexpression promoted cell proliferation of KATO III cells, while the effect was abrogated by silencing MAPK1 expression with transfection of MAPK1 siRNA (Figure 3A). To further investigate the growth promotion mechanisms of GAPLINC on GC cells, we monitored changes in the cell cycle distribution by flow cytometry. Our results showed that overexpression of GAPLINC in KATO III significantly 

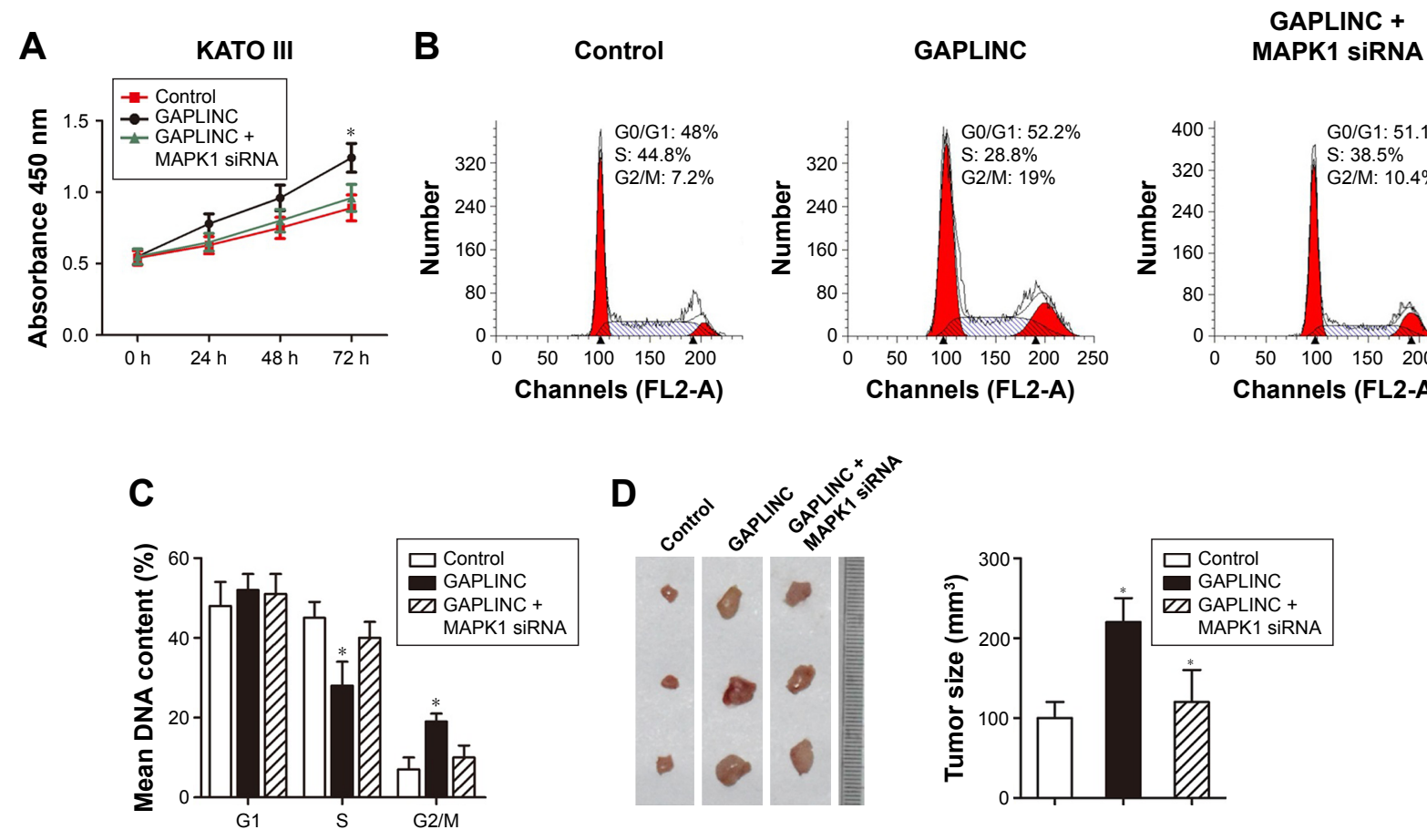

Figure 3 The enhancement of GC cell proliferation and cell cycle progression by IncRNA GAPLINC was dependent on MAPKI. GC cell line KATO III was transfected with control, pCDNA-GAPLINC, or PCDNA-GAPLINC + MAPKI siRNA. (A) Cell proliferation was measured by CCK-8 assay at the indicated times in GC cell line KATO III. Results are represented as the mean \pm SD. $n=5$, ANOVA, $* P<0.05$. (B) Cells were stained with propidium iodide (with RNase A). Cell cycle assay was measured using flow cytometry. Results are represented by three independent experiments. (C) The data of cell cycle were analyzed using Modifit software. Results are represented as the mean \pm SD. $n=3$, ANOVA, $* P<0.05$. (D) KATO III cells were transfected with control, pCDNA-GAPLINC, or PCDNA-GAPLINC + MAPKI siRNA. The cells (I05) were injected subcutaneously into the subcutis on the right flank of nude mice. Surgical resections of KATO III xenograft tumors on week 4 for animals and measurements of tumor volumes are shown. Results are represented as the mean \pm SD. $n=5$ per group, ANOVA, $* P<0.05$.

Abbreviations: GC, gastric cancer; IncRNA, long noncoding RNA; CCK-8, cell counting kit-8.

increased the cell population in $\mathrm{G} 2 / \mathrm{M}$ phase, indicating that GAPLINC could accelerate the cell cycle of GC cells; however, the effect was also abrogated by silencing MAPK1 expression with transfection of MAPK1 siRNA (Figure 3B and $C$ ). Furthermore, we assessed the tumor growth of xenografts derived from KATO III cells that were transfected with pCDNA-GAPLINC or pCDNA-GAPLINC + MAPK1 siRNA prior to subcutaneous injection into nude mice. Our results showed that overexpression of GAPLINC enhanced tumor growth of the KATO III xenografts, while tumor growth enhancement was retarded with silencing of MAPK1 in KATO III GC cell xenografts (Figure 3D). Taken together, overexpression of GAPLINC in GC cells accelerated cell cycle progression by upregulating the MAPK1 expression, thereby influencing the cell growth in vitro and in vivo.

\section{LncRNA GAPLINC acted as a direct target of miR-378 in GC cells}

The ceRNA hypothesis presumes that specific IncRNA can act as sinks for pools of active miRNAs, functionally liberating mRNA transcripts targeted by that set of miRNAs. ${ }^{9}$ Additionally, previous studies have shown that miR-378 can directly target MAPK1 and act as tumor suppressors in GC. ${ }^{13}$ In our study, we found that GC tumor tissues exhibited a significantly lower miR-378 expression compared with para-tumor tissues ( $\mathrm{n}=53, P<0.05$; Figure 4A). Pearson's correlation analysis suggested an inverse relationship between miR-378 and GAPLINC expression in GC tumor tissues ( $\mathrm{n}=30, r=$ $-0.504, P=0.0039$; Figure 4B). Furthermore, we performed a search for miRNAs that have complementary base pairing with GAPLINC, using online software program RNAhybrid (https://bibiserv.cebitec.uni-bielefeld.de/rnahybrid/). The results demonstrated that miR-378 could form complementary base pairing with GAPLINC (Figure 4C). We also observed the expression levels of miR-378 and GAPLINC in three human GC cell lines: KATO III, HGC-27, and SNU-1. The results confirmed a significant negative correlation between miR-378 and GAPLINC in GC cell lines (Figure 4D and E). To confirm the direct relationship between miR-378 and GAPLINC, we constructed luciferase reporter vectors with wild-type GAPLINC sequence and GAPLINC sequence with miR-378 binding site deletion mutation. The luciferase activity was detected in SNU-1 cells by co-transfecting with pmiRGLOGAPLINC-WT and miR-378 mimics, pmiRGLO-GAPLINCMUT and miR-378 mimics, or negative control. The results clearly indicated that miR-378 mimics significantly suppressed 


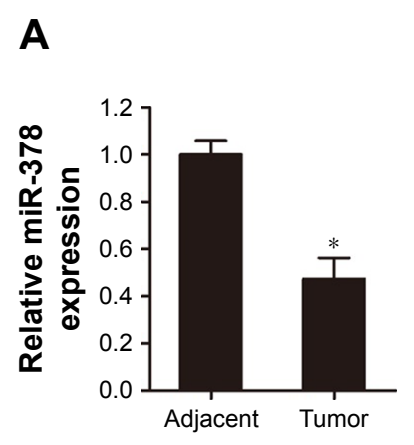

B

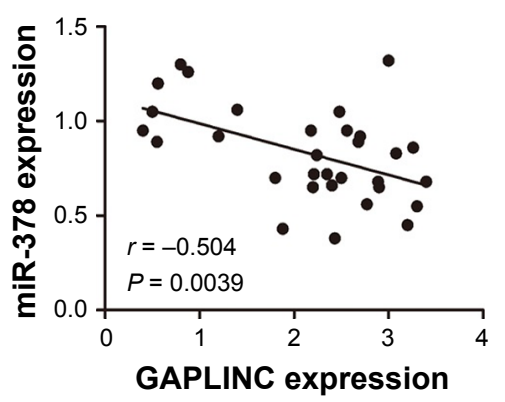

C

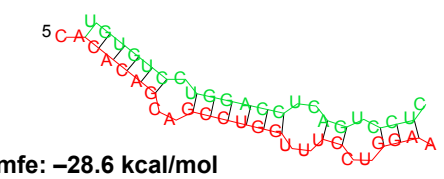

Position: 114

WT-GAPLINC 5 C CA UU CU A3

ACACAG GCCUGG UC GGA

UGUGUC UGGACC AG CCU

miR-378 3 C UC U C 5

MUT-GAPLINC 5 ACACAG GCC $\overline{A C C ~ A G ~ C C U ~} 3$
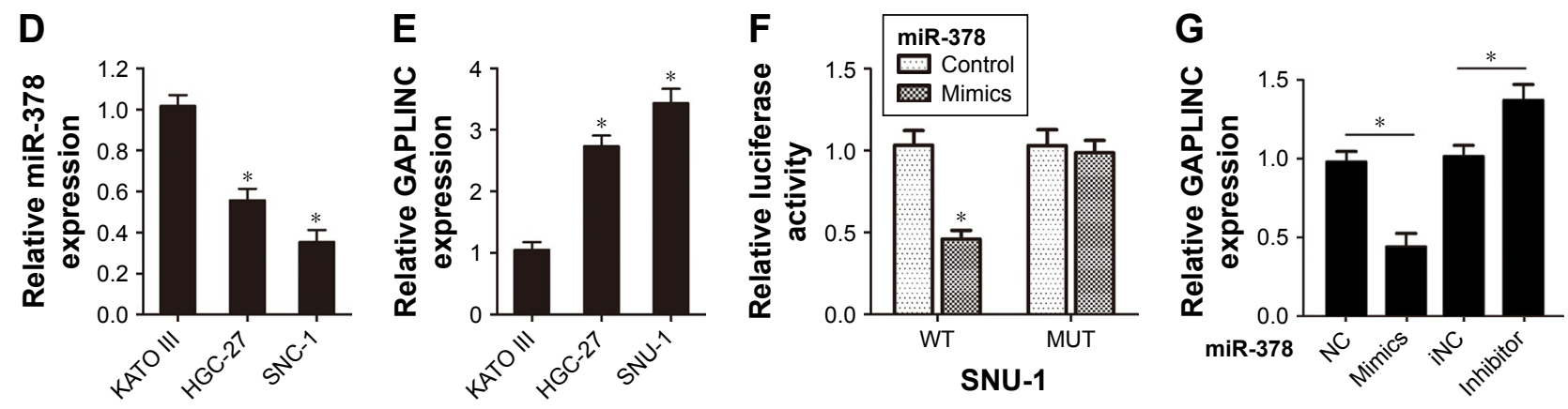

Figure 4 LncRNA GAPLINC acted as a direct target of miR-378 in GC cells. (A) Relative expression of miR-378 in GC tumor tissues was analyzed by real-time PCR. The expression level of $U 6$ was used as control. Results are represented as the mean \pm SD. $n=53$, Student's $t$-test, $* P<0.05$. (B) Pearson's correlation analysis showed a significant negative correlation between IncRNA GAPLINC and miR-378 in GC tissues ( $\mathrm{n}=30, r=-0.504, P=0.0039)$. (C) The binding site of miR-378 on GAPLINC was predicted by RNAhybrid software. (D and E) Relative GAPLINC and miR-378 expression was analyzed by real-time PCR in GC cell lines (KATO III, HGC-27, and SNU-I). Results are represented as the mean \pm SD. $n=3$, ANOVA, $* P<0.05$. (F) Luciferase activity of the indicated group in SNU-I cells after transfected with miR-378 mimics + pmiRGLO vector, miR-378 mimics + pmiRGLO-GAPLINC-WT, or miR-378 mimics + pmiRGLO-GAPLINC-MUT. Results are represented as the mean \pm SD. $\mathrm{n}=5$, ANOVA, $* P<0.05$. (G) Relative GAPLINC expression was analyzed by real-time PCR after SNU-I cells were transfected with control, miR-378 mimics, or inhibitor. Results are represented as the mean \pm SD. $n=3$, ANOVA, $* P<0.05$

Abbreviations: IncRNA, long noncoding RNA; GC, gastric cancer; NC, negative control; iNC, inhibitor negative control.

the activity of pmiRGLO-GAPLINC-WT, but had no effect on the activity of pmiRGLO-GAPLINC-MUT (Figure 4F). Moreover, miR-378 mimics significantly decreased GAPLINC expression and miR-378 inhibitor increased GAPLINC expression in GC cell line SNU-1 (Figure 4G). The data might illuminate that lncRNA GAPLINC could be a direct target of miR-378 in GC cells.

\section{LncRNA GAPLINC acted as a molecular sponge of miR-378 in GC cells}

To confirm that GAPLINC may function as a ceRNA to regulate the expression and effects of MAPK1 on GC cells through competing with miR-378, we transfected miR-378 mimics or miR-378 mimics + pCDNA-GAPLINC in SNU-1 cells. The CCK-8 assay showed that miR-378 overexpression inhibited proliferation of SNU-1 cells, while the effect was abrogated by upregulating GAPLINC expression with transfection of pCDNA-GAPLINC (Figure 5A). Additionally, we monitored changes in the cell cycle distribution by flow cytometry. The results showed that overexpression of GAPLINC in KATO III significantly decreased the cell population in $\mathrm{G} 2 / \mathrm{M}$ phase, indicating that miR-378 could delay the cell cycle of GC cells; however, the effect was also abrogated by upregulating GAPLINC expression (Figure 5B and C). Moreover, GAPLINC overexpression reversed the suppression effects of miR-378 on MAPK1 expression in GC cell line SNU-1 (Figure 5D and E).

These observations suggested that IncRNA GAPLINC enhanced GC cell proliferation in part by acting as a molecular sponge and competitively binding miR-378, thus promoting MAPK1 expression and functions.

\section{Discussion}

Recent evidence of the roles of noncoding RNAs (ncRNAs) in cancer pathogenesis has added to our understanding of the biology of this disease, ${ }^{10,14-16}$ and a growing number of recent papers have revealed that $\operatorname{lncRNAs}$ could compete with miRNA response elements (MREs) of mRNA, thus modulating the expression of target genes. ${ }^{17}$ However, the exact role of miRNA and lncRNA interactions in the tumorigenesis of GC remains 

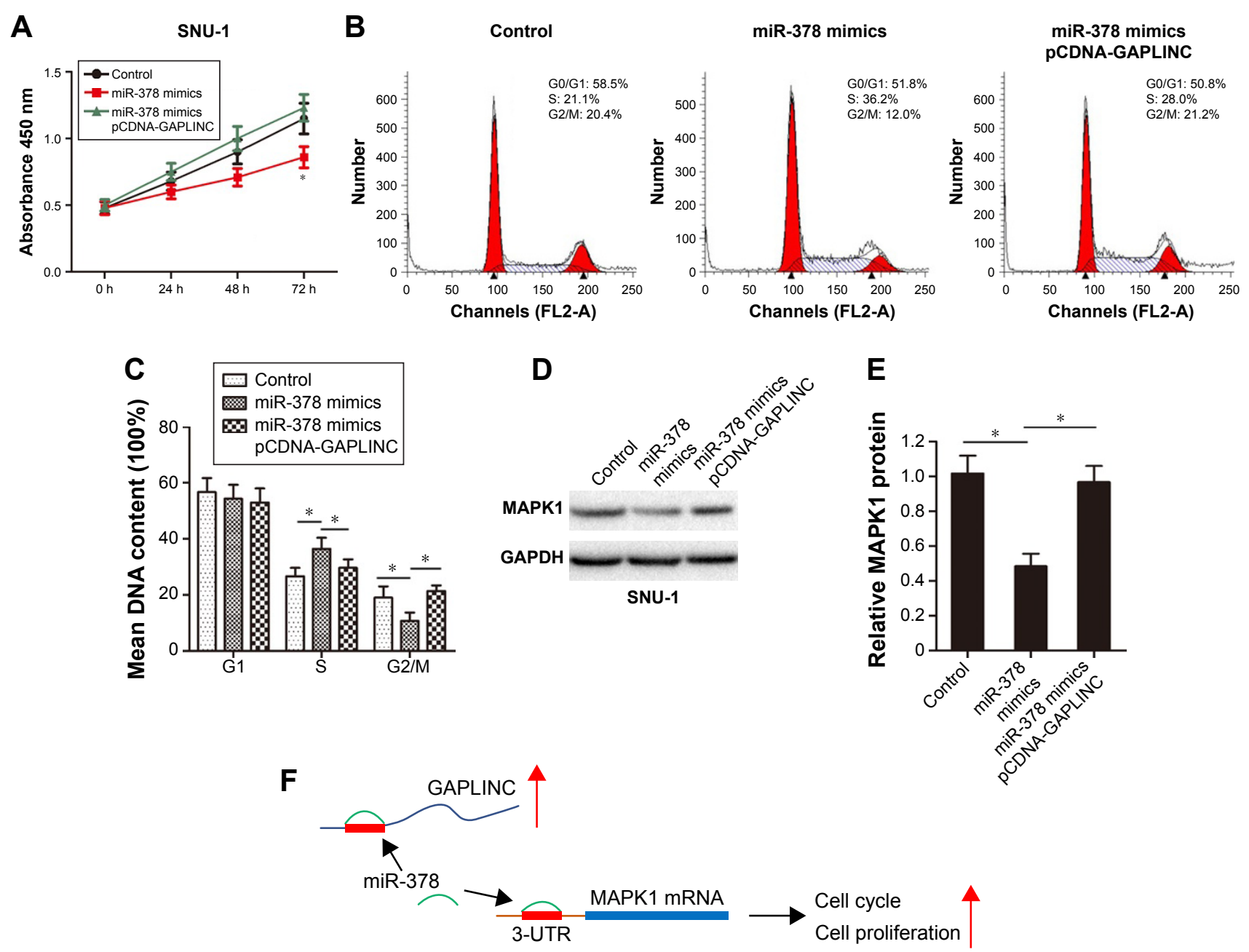

Figure 5 LncRNA GAPLINC acted as a molecular sponge of miR-378 in GC cells. SNU-I cells were transfected with control, miR-378 mimics, or miR-378 mimics + PCDNA-GAPLINC. (A) Cell proliferation was measured by CCK-8 assay at the indicated times in SNU-I cells. Results are represented as the mean \pm SD. $n=4$, ANOVA, $* P<0.05$. (B) Cells were stained with propidium iodide (with RNase A). Cell cycle assay was measured using flow cytometry. Results are represented by three independent experiments. (C) The data of cell cycle were analyzed using Modifit software. Results are represented as the mean \pm SD. $n=3, A N O V A, * P<0.05$. (D) The protein level of MAPKI was analyzed by Western blotting assay. Results are represented by three independent experiments. (E) Quantitative analysis of MAPKI protein level. Results are represented as the mean \pm SD. $n=3$, ANOVA, $* P<0.05$. (F) The proposed signaling pathway underlying GAPLINC regulation of MAPKI by miR-378 in GC cells.

Abbreviations: IncRNA, long noncoding RNA; GC, gastric cancer.

largely unknown. Understanding the interaction between miRNA and IncRNA would facilitate the development of miRNA/ IncRNA-based diagnostics and therapeutics against cancers.

GAPLINC, a newly identified 924-bp lncRNA, was predicted to contain MREs in the promoter region by DataBase of Transcriptional Start Sites. ${ }^{18}$ It is deregulated in GC and associated with copy number variations or oncogenic transcription factors. GAPLINC regulates CD44 as a molecular sponge for miR-211-3p and enhances tumor migration and invasion. ${ }^{18}$ GAPLINC is therefore considered valuable for the diagnosis and prognosis prediction of GC. In the present study, we examined the expression of IncRNA GAPLINC and MAPK1 in GC tissues and cells. The results showed that GAPLINC was upregulated and positively correlated with MAPK1 expression in GC tissues and cells. Additionally, lncRNA
GAPLINC promoted the expression of MAPK1 in GC cells. Additionally, GAPLINC overexpression promoted gastric cell proliferation, increased the cell population in $\mathrm{G} 2 / \mathrm{M}$ cell cycle phase in vitro, and enhanced GC tumor growth in vivo. Moreover, our study further demonstrated that the enhancement of GC cell proliferation and cell cycle progression by lncRNA GAPLINC was dependent on MAPK1.

Additionally, previous studies have shown that miR-378 can directly target MAPK1 and act as tumor suppressors in GC. ${ }^{13}$ Consistent with these studies, we also found that miR-378 could suppress MAPK1 expression, inhibit GC cell proliferation, and delay the cell cycle, suggesting that miR-378 prevents GC cell growth via targeting MAPK1. Inspired by the "competitive endogenous RNAs" regulatory network and emerging evidence that suggests that ncRNAs may participate 
in this regulatory circuitry, ${ }^{19}$ we hypothesized that GAPLINC might also serve as ceRNAs of miR-378 in GC. Therefore, we employed bioinformatics analysis and luciferase assays to confirm the association of GAPLINC and miR-378. As expected, we found miR-378 significantly suppressed the activity of pmiRGLO-GAPLINC reporter vector. Taken together, these data were consistent with our hypothesis and indicated that lncRNA GAPLINC was a direct target of miR-378 in GC cells. Next, we further confirmed the effects of GAPLINC and miR378 on the expression and functions of MAPK1 expression by upregulating GAPLINC in miR-378-overexpressed GC cells. The results showed that GAPLINC reversed the suppression effects of miR-378 mimics on the expression and functions of MAPK1 in GC cells. These observations suggested that GAPLINC promoted GC cell growth in part by competitively binding miR-378 (Figure 5F). Thus, GAPLINC may be a useful biomarker for GC diagnosis and targeted therapy; however, further studies with larger sample size are needed to verify the results. Additionally, the potential mechanism of MAPK1 regulation by GAPLINC should be further analyzed.

\section{Conclusion}

In this study, we demonstrated that there was a significant positive correlation between lncRNA GAPLINC and MAPK1 in GC tissues. Additionally, the enhancement of GC cell proliferation and cell cycle progression by lncRNA GAPLINC was dependent on MAPK1. We also showed for the first time that IncRNA GAPLINC acted as a molecular sponge of miR-378, thus promoting MAPK1 expression. Taken together, the identification of the effects of GAPLINC on GC will improve our knowledge of ncRNAs function, allowing us to better understand the pathogenesis of GC.

\section{Acknowledgment}

This work was supported by Research Center of Nanjing University of Chinese Medicine.

\section{Disclosure}

The authors report no conflicts of interest in this work.

\section{References}

1. Kim EJ, Baik GH. [Review on gastric mucosal microbiota profiling differences in patients with chronic gastritis, intestinal metaplasia, and gastric cancer]. Korean J Gastroenterol. 2014;64(6):390-393. Korean [with English abstract].

2. Kong X, Qian J, Chen LS, et al. Synbindin in extracellular signalregulated protein kinase spatial regulation and gastric cancer aggressiveness. J Natl Cancer Inst. 2013;105(22):1738-1749.

3. Ma Q, Reeves JH, Liberles DA, et al. A phylogenetic model for understanding the effect of gene duplication on cancer progression. Nucleic Acids Res. 2014;42(5):2870-2878.

4. Dwivedi GR, Sharma E, Rao DN. Helicobacter pylori DprA alleviates restriction barrier for incoming DNA. Nucleic Acids Res. 2013;41(5):3274-3288

5. Ota M, Horiguchi M, Fang V, et al. Genetic suppression of inflammation blocks the tumor-promoting effects of TGF-beta in gastric tissue. Cancer Res. 2014;74(9):2642-2651.

6. Wang Y, Wen M, Kwon Y, et al. CUL4A induces epithelial-mesenchymal transition and promotes cancer metastasis by regulating ZEB1 expression. Cancer Res. 2014;74(2):520-531.

7. Qian J, Kong X, Deng N, et al. OCT1 is a determinant of synbindinrelated ERK signalling with independent prognostic significance in gastric cancer. Gut. 2015;64(1):37-48.

8. Fang XY, Pan HF, Leng RX, et al. Long noncoding RNAs: novel insights into gastric cancer. Cancer Lett. 2015;356(2 Pt B):357-366.

9. Salmena L, Poliseno L, Tay Y, et al. A ceRNA hypothesis: the Rosetta Stone of a hidden RNA language? Cell. 2011;146(3):353-358.

10. Chou J, Wang B, Zheng T, et al. MALAT1 induced migration and invasion of human breast cancer cells by competitively binding miR-1 with cdc42. Biochem Biophys Res Commun. 2016;472(1):262-269.

11. Hu CE, Du PZ, Zhang HD, et al. Long noncoding RNA CRNDE promotes proliferation of gastric cancer cells by targeting miR-145. Cell Physiol Biochem. 2017;42(1):13-21.

12. Mao Y, Liu R, Zhou H, et al. Transcriptome analysis of miRNAlncRNA-mRNA interactions in the malignant transformation process of gastric cancer initiation. Cancer Gene Ther. 2017;24(6):267-275.

13. Fei B, Wu H. MiR-378 inhibits progression of human gastric cancer MGC-803 cells by targeting MAPK1 in vitro. Oncol Res. 2012; 20(12):557-564.

14. Li S, Zhang H, Ning T, et al. MiR-520b/e regulates proliferation and migration by simultaneously targeting EGFR in gastric cancer. Cell Physiol Biochem. 2016;40(6):1303-1315.

15. Zhu P, Zhang J, Zhu J, et al. MiR-429 induces gastric carcinoma cell apoptosis through Bcl-2. Cell Physiol Biochem. 2015;37(4): 1572-1580.

16. Zhang Z, Zhu Z, Watabe K, et al. Negative regulation of lncRNA GAS5 by miR-21. Cell Death Differ. 2013;20(11):1558-1568.

17. Shi $X$, Sun M, Liu H, et al. Long non-coding RNAs: a new frontier in the study of human diseases. Cancer Lett. 2013;339(2):159-166.

18. Hu Y, Wang J, Qian J, et al. Long noncoding RNA GAPLINC regulates CD44-dependent cell invasiveness and associates with poor prognosis of gastric cancer. Cancer Res. 2014;74(23):6890-6902.

19. Zhou X, Ye F, Yin C, et al. The interaction between MiR-141 and lncRNA-H19 in regulating cell proliferation and migration in gastric cancer. Cell Physiol Biochem. 2015;36(4):1440-1452.
OncoTargets and Therapy

\section{Publish your work in this journal}

OncoTargets and Therapy is an international, peer-reviewed, open access journal focusing on the pathological basis of all cancers, potential targets for therapy and treatment protocols employed to improve the management of cancer patients. The journal also focuses on the impact of management programs and new therapeutic agents and protocols on

\section{Dovepress}

patient perspectives such as quality of life, adherence and satisfaction. The manuscript management system is completely online and includes a very quick and fair peer-review system, which is all easy to use. Visit http://www.dovepress.com/testimonials.php to read real quotes from published authors. 